\title{
Real-Time Internet of Things Enabled Dashboard for Next Generation Anxiety Risk Classification
}

\author{
Shama Siddiqui, Farid Nait-Abdesselam, Anwar Ahmed Khan, Shamsul Arfin Qasmi and Indrakshi Dey
}

\begin{abstract}
The ubiquity of sensor technology and the Internet of Things prompted us to propose to develop an end-to-end communication architecture for real-time digital dashboards to visualize the anxiety risks of a population during a pandemic, as in the case of COVID-19. Such an architecture can be regarded as the next-generation anxiety risk classification mean for the healthcare industry 4.0 as it will be capable of generating automated and quick actions through the use of analytics on the collected data and predefined thresholds. Based on Internet of Things and wearable healthcare sensors, the proposed end-to-end communication architecture is capable of detecting physiological data related to heart rate, blood pressure, and $\mathrm{SPO}_{2}$, and communicate them to remote cloud servers. Based on this collected data, the centralized dashboard will classify in real time the patients of each geographic region involved according to a specific attribute, namely: normal, mild, moderate, high, severe, or extreme. In addition, we also propose to incorporate the emerging technologies of Space Time Frequency Spreading (STFS) and Space-Time Spreading-Aided Indexed Modulation (STS-IM) for the design of the communication links. It has been found that the integration of STFS and STS-IM promises to reduce the likelihood of data disruption for the proposed architecture.
\end{abstract}

Index Terms-Internet of things, healthcare industry, environmental sensors, real-time dashboards, smart healthcare.

\section{INTRODUCTION}

C OVID-19 has generated a severe crisis for the general healthcare industry worldwide. The healthcare authorities have been overburdened with recruiting, deploying, training and retaining their staff who could serve COVID-19 patients while protecting themselves [1]. At the same time, it has become one of the crucial challenges to timely detect serious health risks among populations and provide critical healthcare services to those in need. In addition to the physiological symptoms of COVID-19, mental health issues have also been reported to rise at an alarming rate during the pandemic. Fear and anxiety about a new illness and what might happen can be overwhelming and cause strong emotions in adults and children. Additionally, public health actions, such as social distancing, reduced physical activity, unpredictability, and routine changes, can all contribute to increasing this stress [2].

S. Siddiqui is with the Department of Computer Science, DHA Suffa University, Karachi, Pakistan, Email: shamasid@ hotmail.com

F. Nait-Abdesselam is with The University of Missouri Kansas City, USA, Email:naf@umkc.edu

A. A. Khan is with Department of Computer Science, Institute of Business Administration, Karachi, Pakistan, Email: yrawna@yahoo.com

S.A. Qasmi is with Department of Pathology/ Microbiology, Fazaia Ruth Pfau Medical College, Faisal Base, Karachi, Pakistan. Email: saqqasmi123@gmail.com

I. Dey is with National University of Ireland and Trinity College Dublin, Ireland, Email: deyi@tcd.ie
People, with or without existing mental health needs, may be distressed by this impact, including with daily living support, continuing care arrangements, support with medication, and changes in their daily routines. For example, shortly after the outbreak of the COVID-19 virus, the anxious behavior of people around the world caused a severe shortage of masks and hand sanitizing solutions [3]. People who already receive services for their mental health, learning disabilities or autism, are even more vulnerable and suffer from increased feelings of panic, stress and anxiety [4]. Therefore, managing anxiety among the world's population is a paramount must during such difficult times.

We believe that geographic location has a predominant impact on anxiety. Although rarely discussed, research in recent years has focused on the intersection between localization and common anxiety disorders [5]. For example, general anxiety disorder is quite common in the southern and western United States, while social phobia is more prevalent in the northeast and mid-west of the country [6]. Likewise, the geographical location has a substantial impact on the stress of minorities. For example, minority stress disorder is quite common among the Coptic Christian population in Egypt and in the Lesbian-Gay-Bisexual-Transgender (LGBT) community among African Americans. Therefore, anxiety about the pandemic, while not naturally apparent, is deeply affected by the geographic location. Another example is the very high death rate caused by the coronavirus that has been recorded in senior housing in Canada. Moreover, the greatest number of pandemic-related anxiety disorder cases has been reported in regions of Canada where the concentration of residences for the elderly or people living in the aging population is the highest. A similar situation has occurred in places and cities across the United States, where more cases of stressrelated disorders have been reported where the majority of the population is believed to be vulnerable to infection with COVID-19 [6].

Collecting and managing demographic data has emerged as a crucial requirement during a pandemic like COVID-19. Industry 4.0 technologies such as Internet of Things (IoT) have been widely used for communicating population's data to the healthcare authorities [7]. In this regard, having automated and real-time dashboards will provide an excellent opportunity for medical experts and state authorities to monitor the health status of populations while maintaining the requirement for social distancing. For example, proper visualization of demographic data not only helps to plan medical facilities in advance, but also helps authorities know the mental health status of the population. Measurement and analysis of some 
vital parameters, such as heart rate and $\mathrm{SPO}_{2}$, can provide a very good insight in real time.

Previously, the major focus of healthcare IoT has been on primarily providing remote monitoring facility to patients individually [8]. However, the data collection from multiple patients and the real-time categorization of the population for certain health risks were not investigated. Additionally, most of the dashboards developed during COVID-19 primarily focused on displaying simple information on the number of diagnosed cases, active cases, severity of cases, and recovered cases from various geographic locations. However, no dashboards or an IoT-based solution have been investigated to categorize populations based on their risk of severe anxiety.

In this article, we present a development of an IoT-based digital dashboard to collect and display real-time data on anxiety risks during a pandemic. We first present the underlying end-to-end communication architecture which facilitates the delivery of the required data, from portable sensors to remote sites for immediate diagnosis and real-time risk analysis of anxiety. In this architecture, we make use of vital parameters, such as heart rate, blood pressure, and $\mathrm{SPO}_{2}$, to assess the level of risk for anxiety, in both COVID-19 patients and other populations, and display them in real time on a digital dashboard. The proposed architecture can be regarded as the next-generation anxiety risk classification mean for the healthcare industry 4.0 as it is capable of generating automated actions through analytics on the collected data and predefined thresholds. In addition, we propose to incorporate the emerging technologies of Space Time Frequency Spreading (STFS) and Space-Time Spreading-Aided Indexed Modulation (STSIM) for the design of the communication links. It has been found that the integration of STFS and STS-IM promises to reduce the likelihood of data disruption in the proposed architecture.

The rest of this paper has been organized as follows: Section II presents a brief review of existing IoT dashboards. Section III describes the proposed architecture and methodology adopted for anxiety risk classification. Section IV presents the experimental hardware design and the obtained experimental results. Section V details the proposed link design and its impacted results. Section VI sheds light on open challenges and future directions for enhancing this area of research. Finally, section VII concludes the article.

\section{EXISTING IOT DASHBOARDS}

Dashboards are the most common forms of human-machine interfaces for an IoT network or other cyber-physical systems. They collect, organize, analyze and transform digital information (data), communicated from the physical world, to represent it in a format that can be easily understood by anyone when read or viewed on a mobile phone, computer or any other user interface [9]. The main advantages of dashboards are their ability to monitor and control input/output devices on a network, can be used for both overall and individual benefits, and can be operated on cloud-based data platforms. Below we present a review of the various dashboards that have been developed over the years and operate using the IoT network.
The first group represents dashboards for general applications and the second presents dashboards specially developed for applications related to pandemic or COVID-19.

\section{A. General Applications}

Water-level monitoring dashboard is developed in [10] to identify and measure the rise in water level in flood prone regions with posting data and alert on Twitter. Air-quality monitoring dashboard is developed in [11] for monitoring and improving air quality in indoor areas based on computed Air Quality Index (AQI). Real-time IoT dashboards are developed in [12] and [13] for animal health monitoring and plant health monitoring and management respectively.

Several dashboards have been developed for monitoring and management of health and lifestyle of individuals remotely [14]-[16]. In [14], data is collected from environmental sensors placed in homes and health-care facilities of monitored individuals for behavioral analysis. Life-style monitoring dashboard is implemented in [15] to make it easier for individuals to track their eating habits and initiate physical activity corresponding to their consumed calories. In [16], data on vital body parameters are collected from a group of wearable physiological sensors, posted and stored on the cloud, displayed on dashboards of smart-phones. All of these dashboards aim at improving the quality of life and healthcare for the patients of diverse population groups, classified based on disease type, health status, etc.

\section{B. Pandemic-related Applications}

There has been a recent surge in the design and development of real-time COVID-19 or pandemic-related IoT dashboards [17]-[19] for monitoring and managing patients, for assisting in situation awareness and critical decision making of healthcare professionals and for helping authorities with relevant information for behavioral analysis and detection across wide regions.

The real-time dashboard of [17] targeted prioritizing emergent, urgent and semi-urgent cases requiring surgery for reducing risk of COVID-19 exposure for surgeons, nurses, technicians and other health-care staff. The focus of the dashboard in [18] is to provide information on the status and location of clinical trials for avoiding any duplication and increasing chances of collaboration for vaccine development. The dashboard developed in [19] populated information about the number of patients tested, test results, availability of beds and ventilators etc. for supporting operational decision making like isolation procedure, etc. An interactive web-based mapping dashboard is developed in [20] for assessing changes in mobility patterns of individuals, and then reporting them over the ArcGIS dashboard. Similarly, visualization dashboards have been developed [21] to help remote consultants, telehealth operators, health management teams and emergency service providers.

It has been seen from the review of recent literature that there has been no dashboard developed as yet which could categorize the anxiety risk for patients. Based on this gap, we propose to develop such a dashboard so the medical 
resources and healthcare service delivery can be planned accordingly. Our proposed framework based on IoT realtime dashboards would facilitate low-latency, energy efficient and high throughput streaming of patient data (recordings of vital parameters) for categorization of individual patients and epicenters/collective geographical areas suffering from anxiety disorders into mild, moderate, elevated, severe and extreme cases. Such a framework enhances remote diagnosis, consultancy and treatment without any diagnostic pressure on the local physicians. In turn, it would reduce the risk of exposure of health-care staffs to patients infected by COVID19 or prone to infection and initiating appropriate automated actions, due to avoiding human intervention.

\section{Architecture And Methodology}

Here, we present a conceptual end-to-end framework that uses the local Wifi and mobile networks to stream vital parameters from the users to the IoT gateway. Based on the data received at the gateway, the monitored population is classified based on their geographical locations into groups at different levels of risk of experiencing anxiety attacks. This categorization is posted on real-time dashboards available to relevant authorities, care-givers and emergency responders, so that necessary action can be taken based both on individual and zonal basis. The framework involves several components and devices built into an architecture and an overall level-tolevel methodology that needs to be followed to initiate apt decision-making.

\section{A. Architecture}

The entire architecture is divided into three parts: a) Userside, b) Gateway and c) Endpoint-side. The user-side part of the architecture consists of a group of non-invasive wearable physiological sensing modules deployed on the body of patient for collecting vital parameters like heart rate, blood pressure and oxygen saturation in blood $\left(\mathrm{SPO}_{2}\right)$. Moreover, a group of environmental sensors are also deployed in the home, workplace (hospitals, nursing homes etc.) for behavioral analysis.

Vital parameters are communicated by sensing nodes to an individual's handheld device, typically a smart phone. The handheld device activates 'Preventive Alerts', if the parameters are outside the predefined threshold, and transmits data for 'contact tracing', to the gateway for alert creation for the consultant physician, audio-video communication, generating alerts for specific actions (like taking pills, doing exercise). At gateway, the parameters are forwarded by the cloud computing platform to the digital dashboard for categorizing population based on their anxiety risk levels. Subsequently, population within each geographical area are assigned one of the six risk levels: normal, mild, moderate, elevated, severe or extreme. Based on the dashboard categorization, response at the endpoint-side is decided. Finally, the endpoint-side involves a) network server for maintaining patient database, b) a group of robots and drones deployed in different risk zones for aiding in services, and c) emergency responders for deployment in severe, extreme and elevated risk zones.

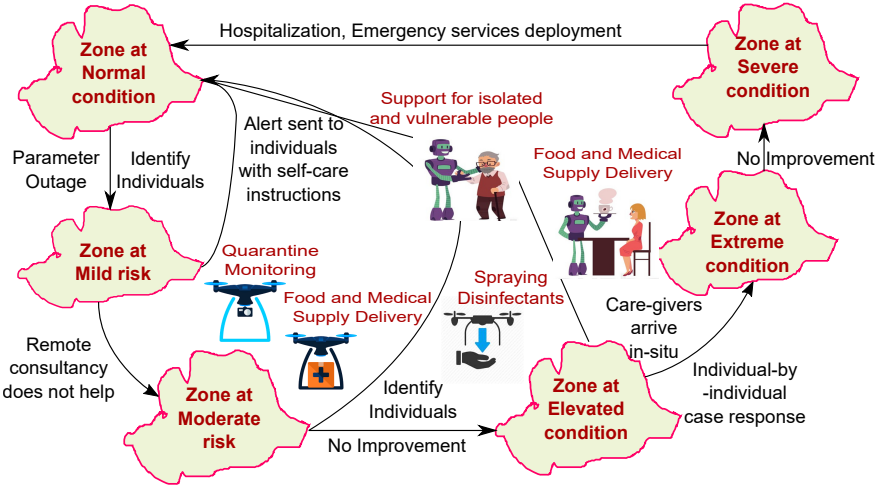

Fig. 1: Schematic diagram of overall methodology based on anxiety risk experienced by different monitored zones and monitored individuals belonging to those zones.

TABLE I: Assigning Risk Levels Using Vital Parameters

\begin{tabular}{|l|l|l|l|l|}
\hline Risk Level & Heart Rate & $\mathrm{SPO}_{2}$ & \multicolumn{2}{|c|}{ Blood Pressure (mmHg) } \\
\cline { 4 - 5 } & $($ Beats/min) & $(\%)$ & Systolic & Diastolic \\
\hline Normal & $60-89$ & $95-100$ & $90-120$ & $60-80$ \\
Mild & $90-100$ & $92-94$ & $121-130$ & $81-84$ \\
Moderate & $100-110$ & $85-92$ & $131-139$ & $85-90$ \\
Elevated & $110-120$ & $75-84$ & $140-159$ & $91-95$ \\
Severe & $120-130$ & $65-74$ & $160-189$ & $96-99$ \\
Extreme & $>130$ & $<65$ & $>190$ & $>100$ \\
\hline
\end{tabular}

\section{B. Methodology}

The overall methodology of the efficient operation of proposed architecture involves a two-stage process. The first stage encompasses categorization of population belonging to different geographical regions into different levels of anxiety risk based on the data about vital parameters streamed in real-time. The second stage involves different measures that are taken once the risk levels at different monitored zones have been identified and segregated using the digital dashboard. The first stage is synonymous to the dashboard operation, while the second stage corresponds to different supporting actions that are to be taken. A schematic diagram of the overall methodology has been detailed in Fig. 1.

The first level is the Normal risk level, where the vital parameters of a geographically-grouped monitored individual is in accordance with his/her age as suggested by their personal physician. No actions or measures are taken for this group of individuals. If a certain group of individuals belonging to a particular area experience slight aberrations in their vital parameters on a daily basis, they are categorized into Mild risk level. Alerts along with appropriate self-care measures like breathing exercises, listening to music or comforting voices, taking a walk, talking to loved ones etc. Alerts are sent to identified individuals within the Mild risk. The alerts are sent intermittently till the parameters are back in the normal range.

If the alerts and self-help measures are not able to bring the body parameters back within the normal range after a certain amount of time, the individuals experiencing this condition within a certain geographical area are grouped into Moderate 
risk level. Individuals belonging to this risk zone may feel more overwhelmed, agitated and nervous. With the pandemic situation in mind, the measures that can be taken are sending drones for rapid delivery of medicines and food supplies, carrying out large-scale temperature measurements through infrared cameras.

Individuals belonging to a wider area who experience body parameters like heart rate, $\mathrm{SPO}_{2}$ and blood pressure are higher than a predefined threshold, but do not need emergency attention, can be grouped into elevated risk zone. Even in this case, with the pandemic scenario in view, drones will be sent out for delivering required supplies. Robots will be sent out for assisting elderly, isolated and vulnerable individuals in their daily work and keeping them company. Robots will also help in regular blood and saliva sample from the individuals for diagnosis of other ailments.

If the situation still does not improve for certain group of individuals, they will identified and grouped into severe risk zone. Individuals belonging to this zone suffer from serious anxiety risk and need medical intervention. Human caregivers will be sent out for individual-by-individual case. Before sending over the caregivers, identified areas will be thoroughly sprinkled by disinfectants from drones and important information will be broadcasted through drones. Finally, the highest risk level extreme which represents a small group of individuals within an area that are experiencing panic attack and need emergency services and may be hospitalized.

\section{Hardware DESign AND EXPERIMENTAl RESUlts}

For experimentally validating the concept of our dashboard, we have developed an initial high-level system prototype involving Node Micro-controller Unit (NodeMCU), Pulse Oximetry Sensor MAX 30100, Electronic Wrist Blood Pressure Monitor CK-101 and I2C OLED module. A schematic diagram for the system-level prototype designed and how it can be incorporated within our proposed anxiety-risk detection framework is demonstrated in Fig. 2. Fig. 2 also includes the schematic diagram of system-level prototype including detailed circuit diagram and pin configuration for the prototype.

\section{A. High-level Prototype}

NodeMCU is in the core of our prototype. It is an open source IoT platform which offers interactive, easy and userfriendly application programming interface (API). It includes firmware that runs on the the ESP8266 wiFi from Espressif system, and ESP-12 module. Pulse Oximetry Sensor MAX 30100 is an integrated solution for capturing the values of both heart rate and $\mathrm{SPO}_{2}$ using photo-detectors. The solution works with the help of two light-emitting diodes (LEDs) emitting red and infrared lights. Infrared light is used to measure the heart rate, whereas both red and infrared lights are used for measuring $\mathrm{SPO}_{2}$. Electronic Wrist Blood Pressure Monitor CK-101 Sphygmomanometer is used for monitoring the Systolic (upper) and Diastolic (lower) blood pressure. CK101 is a handheld monitor equipped with inflatable wristband (with a wrist circumference of $135-195 \mathrm{~mm}$ ). The OLED Module is used to display the message to the user about the heart rate/ $\mathrm{SPO}_{2}$ values along with the risk level. This module provides a display in 2 rows, with each row supporting 16 characters of customized messages.

\section{B. Simulation Results}

For validating the working of the prototype heart rate, $\mathrm{SPO}_{2}$ and blood pressure levels from multiple patients (around 500) belonging to five different geographical regions are collected to determine whether they are normal or at mild, moderate, elevated, severe or extreme risks of anxiety attacks. Data related to vital parameters of patients suffering from previous history of frequent anxiety attacks, chronic disease, high vulnerability to COVID-19 infection and infected with COVID-19 is accessible in public archives (Kaggle; https://www.kaggle.com/datasets and Github; https://github.com/HDRUK/datasets).

Fig. 3 represents a view of central dashboard where the categorization of data collected from a single user has been presented. The Google Sheet was populated by running a Google Script (Gscript) tied to the NodeMCU. 30 samples were collected at an interval of 5 minutes. The same procedure can be repeated for all the users involved and can be displayed simultaneously on the dashboard. The vital parameters are displayed on an OLED display for our initial prototype and are reported also over the cloud (Google Sheet). The collected data in a tabular format, the trends of $\mathrm{SPO}_{2}$, blood pressure, heart rate and a pictorial representation of the digital dashboard, are also demonstrated in Fig. 3. Since, only one user is exhibited in the portrayal of Fig. 3, the dashboard presents his/her risk levels, assuming the user to be located in zone 1 .

Results form 500 users (100 from each zone) are plotted in Fig. 6. From region 1, 24 people have a normal level of $\mathrm{SPO}_{2}$, so they are free of anxiety risk, 14 people have mild and 25 have moderate risk. These risks are also not as alarming and can easily be managed through self-care. However, it has been shown that 12 people have elevated risk, 17 have severe and 8 have extreme risk; for these users, there would be a potential need for providing remote assistance for anxiety management or some other immediate guidance. As can be seen in Fig. 6, zone 5 has the most patients with higher risks followed by zones 2,1, 3 and 4. Similar observations can also be made based on heart rate and blood pressure values, zones 4 and 5 are at most risk of developing anxiety respectively. It is quite evident that zones 4 and 5 need attention and appropriate action needs to be taken based on individual requirements.

\section{LINK DESIGN}

A crucial part of the proposed framework is, i) the design of the communication link between the user's handheld device and the IoT gateway equipped with the cloud computing platform for posting data on the IoT dashboards, as well as, ii) the link between the gateway and the application endpoint that needs to be activated depending on the observation of the dashboard and appropriate action that needs to be taken based on that observation. Here, we are proposing two enabling technologies specifically for each of the links to ensure information delivery with minimum latency and maximum reliability in an energy efficient way. A conceptual diagram 


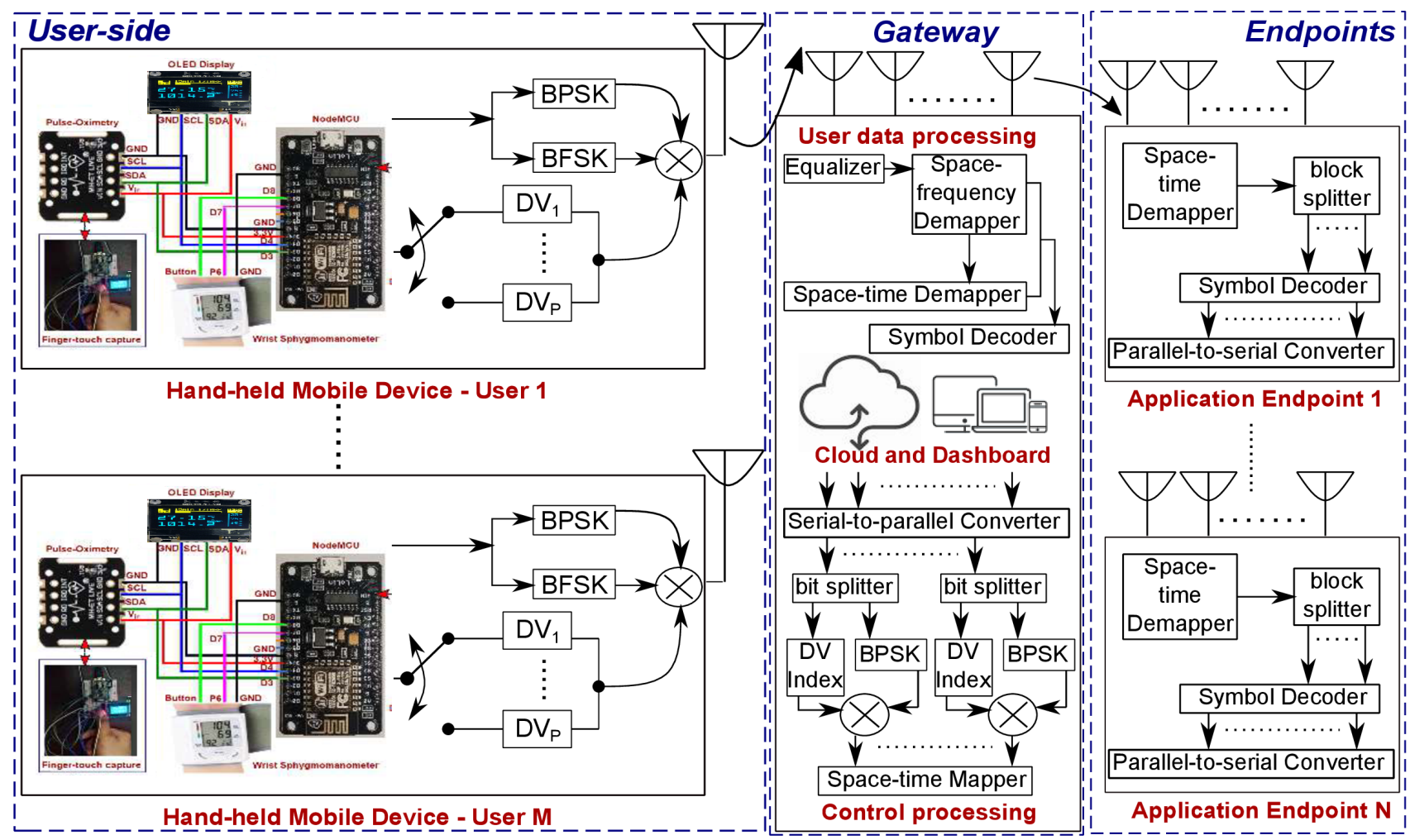

Fig. 2: Conceptual architecture incorporating three entities; the first part is user-side consisting of sensors and hand-held devices, the second part is IoT gateway aided by a cloud computing platform and a cloud powered real-time dashboard; the final part is application endpoints involving network servers, health-care centers etc.

of the communication link design involved with the proposed framework is provided in Fig. 2.

\section{A. Enabling Technologies}

1) User-to-Gateway: Energy efficient transmission of body parameters from the user's hand-held devices to the IoT gateway can be accomplished by reducing the transmission energy required for fighting impairments. The user devices are assumed to be simplistic, equipped with single antenna and the gateway is furnished with multiple antennas. Therefore, patient data can be spread over space-time-frequency blocks before reporting them to the gateway; a technique that can be coined as Space Time Frequency Spreading (STFS).

System Model: The key idea of STFS-aided transmission is to map user information on indexed space-time and spacefrequency blocks of fixed duration and bandwidth respectively, by multiplying individual information vectors with different dispersion matrices. Correspondingly at the gateway, a spacefrequency demapper is implemented for detecting activated frequency sub-bands followed by a space-time demapper for identifying the time slots. Finally, a maximum likelihood (ML) symbol decoder estimates the dispersion matrix and patient data from the demapped signal.

For example, if $M$ users or $M$ hand-held devices are transmitting their information simultaneously over a symbol duration of $\tau$, the transmit vector from the $m$ th user can be expressed as,

$$
\mathbf{d}_{m}^{l}=\operatorname{vec}\left(\mathbf{x}_{m}^{l}{ }^{t}\right) A_{p, m}^{l} \in \mathbb{C}^{\tau \times \tau}
$$

for $(l=1,2, \ldots, L)$, where $\mathbf{x}_{m}^{l}=s_{m}^{l} \mathbf{r}_{m}^{l} \in \mathbb{C}^{1 \times \tau}$ is the information vector from the $m$ th user, $t$ denotes transpose of a matrix, vec represents the vectorial stacking operation, $L$ is the total number of space-time-frequency (STF) blocks. Here $s_{m}^{l} \triangleq\{+1,-1\}$ is the binary phase shift keying (BPSK) modulated signal and $\mathbf{r}_{m}^{l} \triangleq\left\{\cos \left(2 \pi f_{1} \tau\right), \cos \left(2 \pi f_{2} \tau+\pi\right)\right\}$ is binary frequency shift keying (BFSK) modulated symbol and $f_{1}$ and $f_{2}$ are separated by at least $1 / \tau \mathrm{Hz}$ in order to maintain orthogonality. Here, $A_{p, m}^{l}=\left[a_{p, m, 1}^{l}, \ldots, a_{p, m, \tau}^{l}\right] \in$ $\mathbb{C}^{1 \times \tau}$ is the $m$ th row of the $p$ th STF dispersion matrix $\mathbf{A}_{p}^{l}=\left[A_{p, 1}^{l}, \ldots, A_{p, M}^{l}\right]^{t} \in \mathbb{C}^{M \times \tau}$ selected out of the $P \mathrm{STF}$ matrices taken from the set $\left\{\mathbf{A}_{p}^{l}\right\}_{p=1}^{P}$

Transmission Signal Model: The encoded set of transmitted information from multiple user devices $\mathbf{D}^{l} \in \mathbb{C}^{M \times \tau^{2}} \triangleq$ $\left[\mathbf{d}_{1}^{l}, \ldots, \mathbf{d}_{M}^{l}\right]^{t}$ must include STF dispersion matrices (DMs) that satisfy the power constraint of $\operatorname{Tr}\left(\mathbf{A}_{p}^{l H} \mathbf{A}_{p}^{l}\right)=\tau \forall p$ to ensure unity energy over each ST block. If the network server or the destination devices are equipped with $N$ receive antennas, the generic $N \times \tau^{2}$ discrete-time received signal matrix can be given by,

$$
\mathbf{Y}^{l}=\sqrt{\rho^{l}} \mathbf{G}^{l} \mathbf{D}^{l}+\mathbf{w}^{l} \in \mathbb{C}^{N \times \tau^{2}}
$$

where $\mathbf{Y}^{l}, \mathbf{G}^{l} \in \mathbb{C}^{N \times M}$ and $\mathbf{w}^{l}$ are the received signal vector, the channel matrix and the noise vector respectively. The noise vector also accounts for different levels of channel state information (CSI) estimation error, where the estimated channel on the receiver side is contaminated by additive Gaussian noise. The constant $\rho^{l}$ denotes the energy spent by any of the sensors during the reporting phase and $\mathbf{G}^{l}$ includes all the samples of the channel impulse response (CIR) between 


\begin{tabular}{|c|c|c|c|c|c|c|c|}
\hline \multirow{3}{*}{\begin{tabular}{|l} 
S.No \\
1
\end{tabular}} & \multirow[b]{2}{*}{ Time } & \multicolumn{5}{|c|}{ Google Sheet with user data } & \multirow[b]{2}{*}{ Diastoli } \\
\hline & & Heart Rate & $\begin{array}{l}\text { Risk_HR } \\
\end{array}$ & $\mathrm{SPO2}$ & $\begin{array}{l}\text { Risk_SPO } \\
\end{array}$ & Systolic & \\
\hline & $10: 15: 00$ & 97 & Mild & 96 & Normal & 110 & 75 \\
\hline 2 & $10: 20: 00$ & 97 & Mild & 96 & Normal & 110 & 75 \\
\hline 3 & $10: 25: 00$ & 97 & Mild & 96 & Normal & 110 & 75 \\
\hline 4 & $10: 30: 00$ & 97 & Mild & 97 & Normal & 112 & 78 \\
\hline 5 & $10: 35: 00$ & 97 & Mild & 96 & Normal & 112 & 78 \\
\hline 6 & $10: 40: 00$ & 103 & Moderate & 96 & Normal & 112 & 78 \\
\hline 7 & $10: 45: 00$ & 98 & Mild & 97 & Normal & 118 & 79 \\
\hline 8 & $10: 50: 00$ & 103 & Moderate & 96 & Normal & 118 & 79 \\
\hline 9 & $10: 55: 00$ & 103 & Moderate & 96 & Normal & 118 & 79 \\
\hline 10 & $11: 00: 00$ & 103 & Moderate & 97 & Normal & 121 & 81 \\
\hline 11 & $11: 05: 00$ & 103 & Moderate & 96 & Normal & 121 & 81 \\
\hline 12 & $11: 10: 00$ & 103 & Moderate & 96 & Normal & 121 & 81 \\
\hline 13 & $11: 15: 00$ & 103 & Moderate & 97 & Normal & 122 & 82 \\
\hline 14 & $11: 20: 00$ & 103 & Moderate & 96 & Normal & 122 & 82 \\
\hline 15 & $11: 25: 00$ & 103 & Moderate & 96 & Normal & 122 & 82 \\
\hline 16 & $11: 30: 00$ & 103 & Moderate & 97 & Normal & 120 & 76 \\
\hline 17 & $11: 35: 00$ & 103 & Moderate & 96 & Normal & 120 & 76 \\
\hline 18 & $11: 40: 00$ & 103 & Moderate & 96 & Normal & 120 & 76 \\
\hline 19 & $11: 45: 00$ & 103 & Moderate & 97 & Normal & 118 & 73 \\
\hline 20 & $11: 50: 00$ & 103 & Moderate & 96 & Normal & 118 & 73 \\
\hline 21 & $11: 55: 00$ & 105 & Moderate & 96 & Normal & 118 & 73 \\
\hline 22 & $12: 00: 00$ & 105 & Moderate & 97 & Normal & 120 & 76 \\
\hline 23 & $12: 05: 00$ & 105 & Moderate & 97 & Normal & 120 & 76 \\
\hline 24 & $12: 10: 00$ & 105 & Moderate & 97 & Normal & 120 & 76 \\
\hline 25 & $12: 15: 00$ & 103 & Moderate & 97 & Normal & 117 & 80 \\
\hline 26 & $12: 20: 00$ & 103 & Moderate & 97 & Normal & 117 & 80 \\
\hline 27 & $12: 25: 00$ & 103 & Moderate & 97 & Normal & 117 & 80 \\
\hline 28 & $12: 30: 00$ & 103 & Moderate & 97 & Normal & 118 & 78 \\
\hline 29 & $12: 35: 00$ & 103 & Moderate & 97 & Normal & 118 & 78 \\
\hline 30 & $12: 40: 00$ & 103 & Moderate & 97 & Normal & 118 & 78 \\
\hline
\end{tabular}

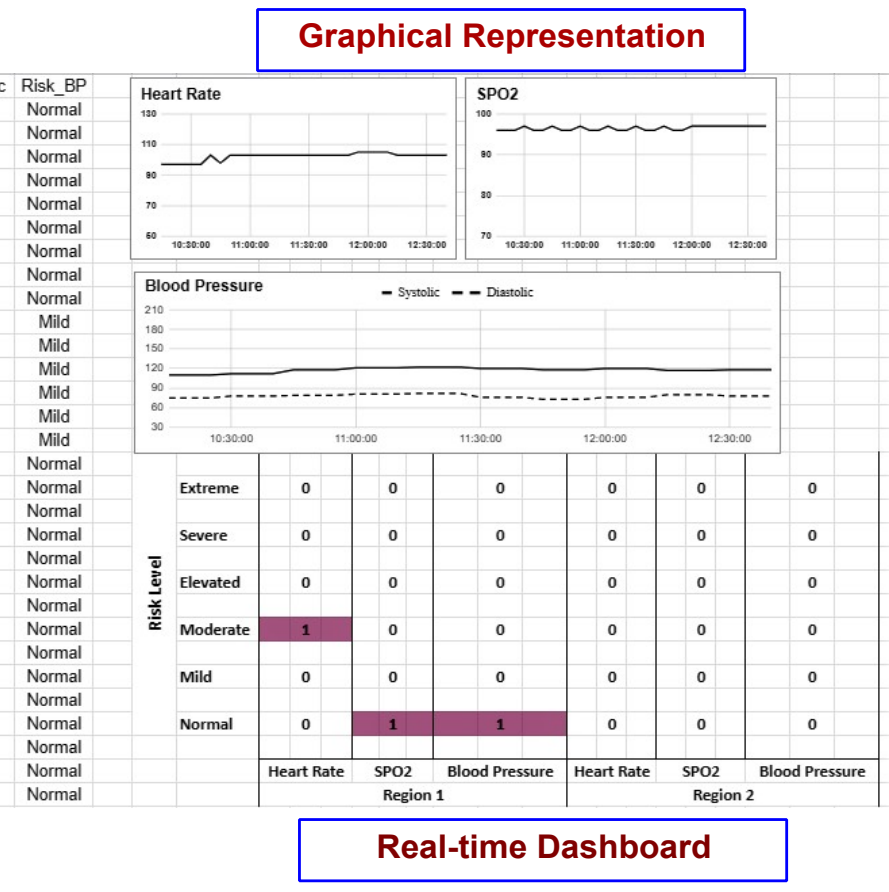

Fig. 3: Real-time Dashboard on Cloud platform powered by Google Sheets with representation of single user data where the user belongs to zone 1. It also depicts the graphical representation of the user data and the detailed sheet of the data samples collected.

the user and the destination devices/network server over the lth STF block.

Reception Signal Model: On the receiver side (network server or devices belonging to the service providers or responders), we will employ an ST demapper, which is essentially vectorization of the received signal. In this case, the linearized equivalent received signal model can be obtained as,

$$
\hat{\mathbf{Y}}^{l}=\sqrt{\rho^{l}} \hat{\mathbf{G}}^{l} \hat{\mathbf{A}}^{l} \hat{\mathbf{\Xi}}^{l}+\hat{\mathbf{w}}^{l}=\operatorname{vec}\left(\mathbf{Y}^{l}\right) \in \mathbb{C}^{N \tau \times 1}
$$

where $\hat{\mathbf{G}}^{l}=\mathbf{I}_{g} \otimes \mathbf{G}^{l} \in \mathbb{C}^{N \tau^{2} \times M \tau^{2}}$ (where $\left.\mathbf{I}_{g}=\mathbf{I}_{\tau^{2} \times \tau^{2}}\right)$ is the estimated channel matrix and $\otimes$ is the Kronecker product, $\hat{\mathbf{w}}^{l}=\operatorname{vec}\left(\mathbf{w}^{l}\right) \in \mathbb{C}^{N \tau^{2} \times 1}, \hat{\mathbf{A}}^{l}=\mathbf{I}_{a} \otimes \mathbf{A}^{l} \in \mathbb{C}^{M \tau^{2} \times M Q}$ (where $\left.\mathbf{I}_{a}=\mathbf{I}_{M \times M}\right)$ and $\hat{\boldsymbol{\Xi}}^{l}=\operatorname{vec}\left(\boldsymbol{\Xi}^{l}\right) \in \mathbb{C}^{M Q \times 1}$ is the received information vector. If the conditional probability the linearized equivalent received signal model (corrupted by noise with a noise spectral density of $N_{0}$ ) is given by

$$
P\left(\hat{\mathbf{Y}}^{l} \mid \hat{\boldsymbol{\Xi}}^{l}\right)=\frac{1}{\pi N_{0}} \exp \left(-\frac{\hat{\mathbf{Y}}^{l}-\sqrt{\rho^{l}} \hat{\mathbf{G}}^{l} \hat{\mathbf{A}}^{l} \hat{\mathbf{\Xi}}^{l}}{N_{0}}\right)
$$

the ML detection criterion is formulated as,

$$
\hat{p}=\underset{p}{\arg \min }|| \hat{\mathbf{Y}}^{l}-\mathbf{x}^{l}\left(\sqrt{\rho^{l}} \hat{\mathbf{G}}^{l} \hat{\mathbf{A}}^{l}\right) \|^{2}
$$

where $\mathrm{x}^{l}$ represents the transmit information vector on the $l$ th STF block. Other sub-optimum detectors like minimum mean-squared error (MMSE) or zero-forcing (ZF) can be used to estimate the dispersion matrix and transmit information vector from $\hat{\boldsymbol{\Xi}}^{l}$. It is worth-mentioning that ML detector is computationally exhaustive and the choice of the dispersion matrix is absolutely crucial for the overall performance; a generalization we will look into in our future work.
Design Criteria for Dispersion Matrices (DM): For the performance analysis, we have chosen $P=M=\tau$. Accordingly, we choose our DM based on random search following the steps:

- Randomly generate $P$ unitary dispersion matrix set $\left\{\mathbf{A}_{p}^{l}\right\}_{p=1}^{P}$ using Gaussian distribution, such that $\operatorname{Tr}\left(\mathbf{A}_{p}^{l H} \mathbf{A}_{p}^{l}\right)=\tau \forall p$.

- The matrix generation is repeated for 10 times.

- For each of the 10 sets of $\left\{\mathbf{A}_{p}^{l}\right\}_{p=1}^{P}$, calculate the corresponding link capacity $C=$,

$$
\frac{1}{\tau}\left(1+\log _{2} P-\frac{1}{2 P} \sum_{p} \mathrm{E}\left[\log _{2}\left\{\sum_{\hat{p}} \exp \left(z_{p}^{\hat{p}}\right) \mid \hat{\boldsymbol{\Xi}}_{\hat{p}}^{l}\right\}\right]\right) .
$$

where $z_{p}^{\hat{p}}=-\left\|\sqrt{\rho^{l}} \hat{\mathbf{G}}^{l} \hat{\mathbf{A}}^{l}\left(\hat{\boldsymbol{\Xi}}_{p}^{l}-\hat{\boldsymbol{\Xi}}_{\hat{p}}^{l}\right)+\hat{\mathbf{w}}^{l}\right\|^{2}+\left\|\hat{\mathbf{w}}^{l}\right\|^{2}$

- Out of the sets of $\left\{\mathbf{A}_{p}^{l}\right\}_{p=1}^{P}$, the set exhibiting the maximum $C$ is chosen for simulating performance.

where $\mathrm{E}[\cdot]$ denotes the expectation and $\|\cdot\|^{2}$ denotes the Frobenius norm.

2) Gateway-to-Endpoint: In order to strike a balance between high reliability and low latency, a combination of spacetime spreading (STS) [22] and indexed modulation (IM) [23] can be implemented at the gateway for the control instruction or activation message that is sent from the gateway to the specific application endpoint to activate a particular service as per requirement. In this case, both the gateway and the application endpoints are equipped with multiple antennas, and the modulation technique can be coined as space-timespreading-aided-indexed-modulation (STS-IM).

Information vectors at the gateway are first converted to parallel sub-blocks of data, each of which are sent over the transmit antennas. Each of the parallel sub-blocks will consist 


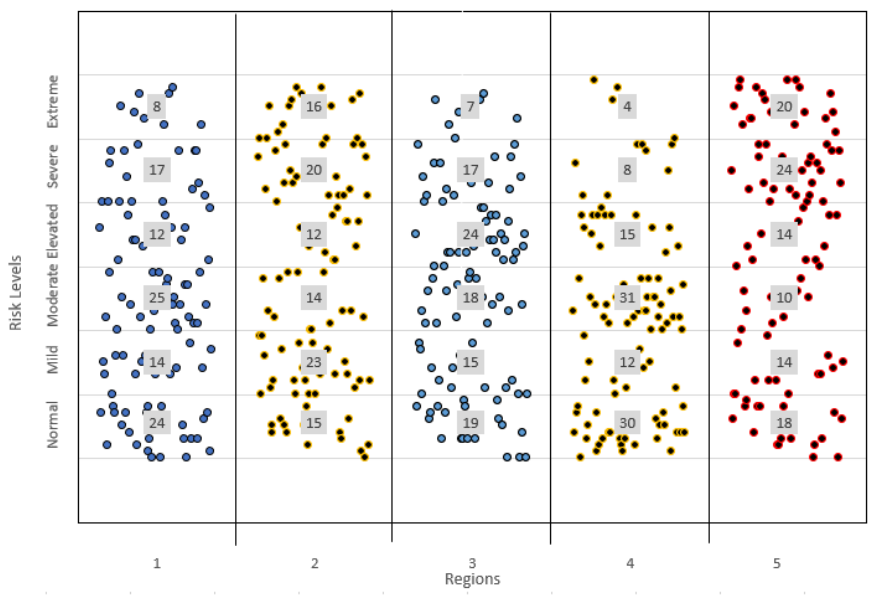

(a)

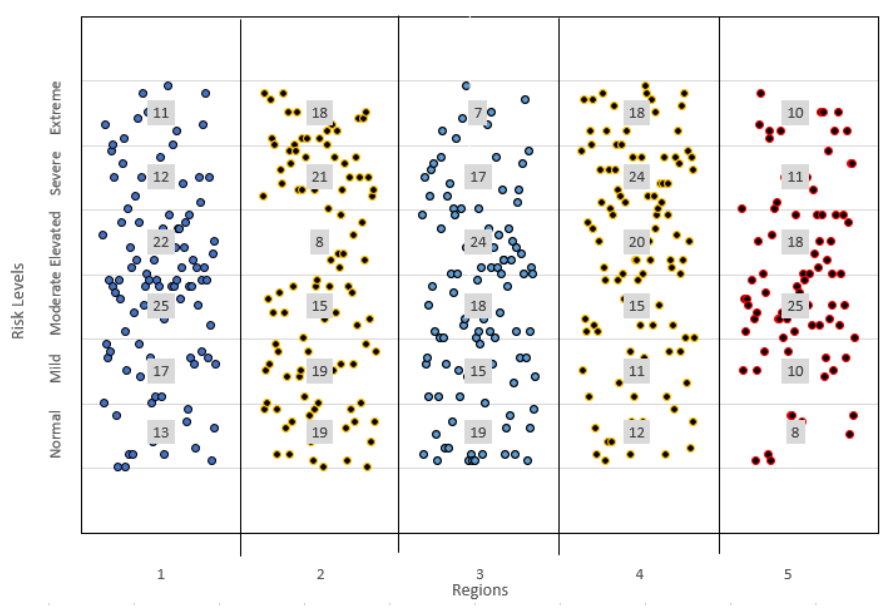

(b)

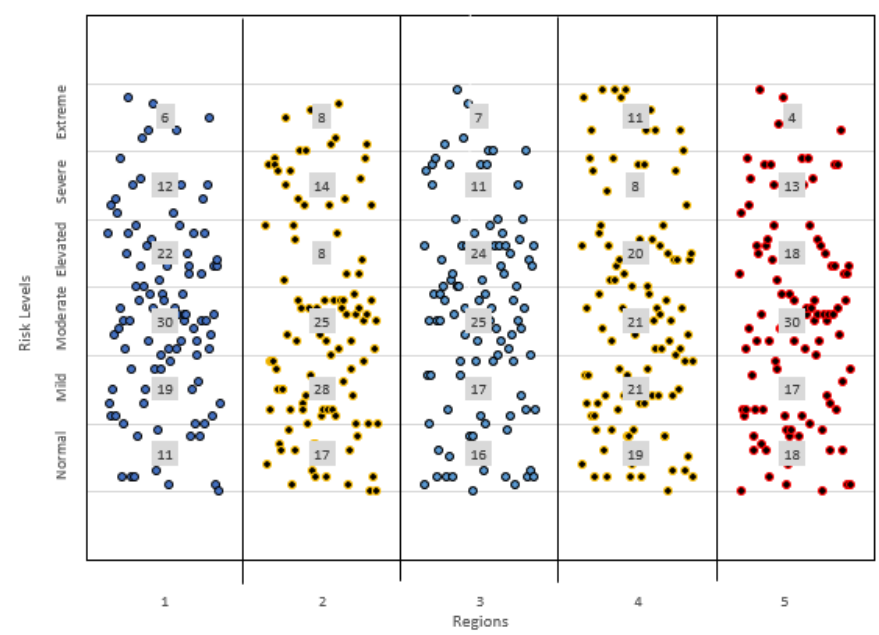

(c)

Fig. 4: Dashboard categorization results for classifying population into different levels of risk experiencing anxiety attacks based $\mathrm{SPO}_{2}$, Heart rate and Blood pressure data.

of two information bits, the first one of which is used to select the index of the dispersion vectors to be activated and the second one is mapped onto information-carrying symbols.

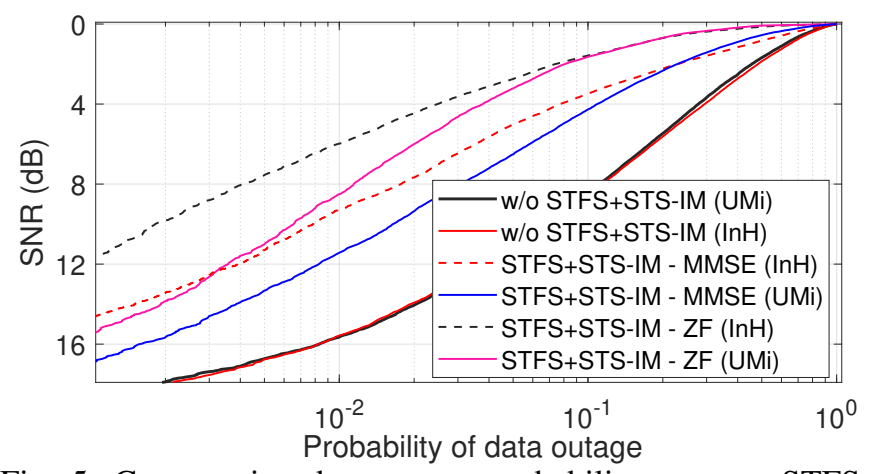

Fig. 5: Comparative data outage probability over an STFSaided user-to-gateway and STS-IM-aided gateway-to-endpoint links.

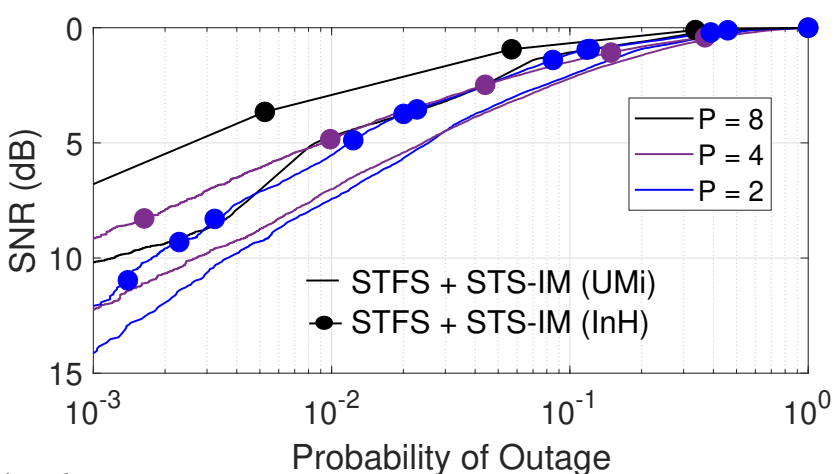

Fig. 6: Variation data outage probability over an STFS-aided userto-gateway and STS-IM-aided gateway-to-endpoint links based on the number of dispersion matrices $P$ available. MIMO-ZF is used as symbol decoder is used at the end of gateway-to-endpoint links.

On the receive side, the data received over multiple antennas are first collected in a space-time demapper to estimate the dispersion vectors. The information symbols extracted after identifying the dispersion vectors are passed through a block splitter followed by a ML/MMSE decoder and a parallel-toserial converter in order to obtain actual control message.

\section{B. Simulation Results}

Next, we simulate the end-to-end outage probability performance of our proposed synthetic IoT-based framework where 10 users (hand-held devices equipped with single antenna) transmit patient data simultaneously. The gateway (32 antennas) is equipped with the cloud computing platform which computes data for ease of visualization through a real-time dashboard, such that appropriate decision can be taken and specific control instruction is activated and transmitted to the relevant endpoint (each with 8 antennas), like network server, deployment centers for UAVs, robots etc. The users are deployed randomly and uniformly distributed in a circular annulus around the IoT gateway. Only one user is activated on a particular space-time-frequency block, when and where all other users are silent.

The channel between the users, gateway and endpoints is assumed to be dispersive with Rayleigh fading envelope and normalized Doppler frequency of $0.01 \mathrm{~Hz}$, while the pathloss is experienced according to the 3GPP Urban Microcell (UMi) model [24] for outdoor links and 3GPP Indoor Hotspot (InH) model [25] for indoor links. Binary Phase Shift 


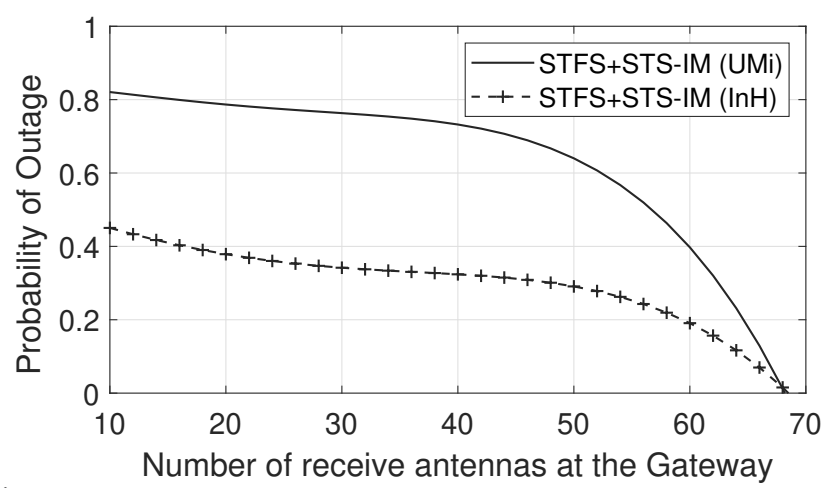

Fig. 7: Variation data outage probability over an STFS-aided user-togateway and STS-IM-aided gateway-to-endpoint links as a function of the number of receive antennas at the IoT Gateway. MIMO-ZF is used as symbol decoder is used at the end of gateway-to-endpoint links. The overall link SNR is fixed at $15 \mathrm{~dB}$.

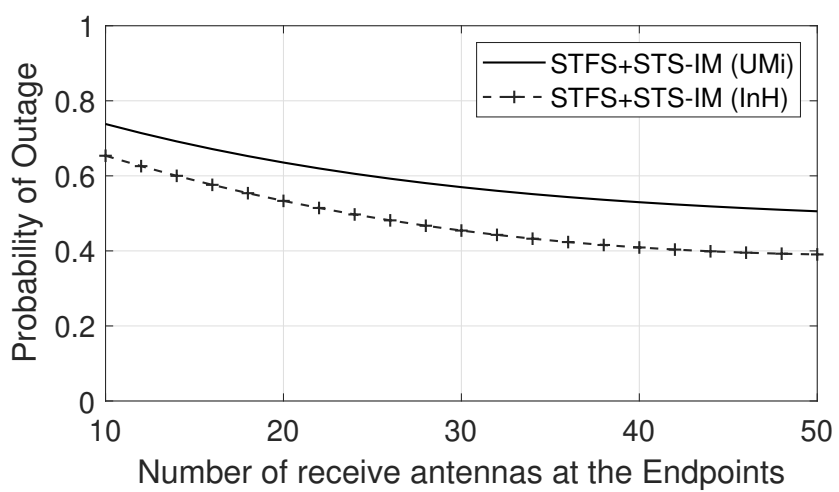

Fig. 8: Variation data outage probability over an STFS-aided user-togateway and STS-IM-aided gateway-to-endpoint links as a function of the number of receive antennas at the application endpoint. MIMO-ZF is used as symbol decoder is used at the end of gateway-to-endpoint links. The overall link SNR is fixed at $15 \mathrm{~dB}$.

Keying (BPSK), Binary Frequency Shift Keying (BFSK) and two space-time-frequency slots on the STFS side and only BPSK and two space-time slots on the STS-IM side with four dispersion vectors to choose form in each case. Our proposed link design offers significant reduction in outage probability in comparison to the network without STFS and STS-IM, as is evident in Fig. 5. Hence it has been established that using MIMO-ZF based symbol decoders at the application endpoint offer better performance over other decoders.

An improvement in performance is observed when 3GPP $\mathrm{InH}$ model is used for modelling the links between the user, gateway and the endpoints, as compared to the 3GPP UMi link modelling. The choice for the InH model can be justified for the scenario where the users are located in their homes or any other indoor environment and the endpoints like the consultant, caregiver, responder etc. are also located in a health-care center or any other indoor settings. The UMi model is used for depicting the scenario where the users and the endpoints are located outdoors. A mixture of indoor and outdoor scenarios is also possible to encounter.

The curves in Fig. 6 characterizes the outage probability, comparing the effects of the number of the dispersion matrices $P$. For this set of curves, MIMO-ZF is used as the symbol decoder at the endpoint side. The set-up architecture is kept same with 10 users, gateway with 32 antennas and an application endpoint with 8 antennas. It is evident that more the choice and flexibility we have in terms of the dispersion matrices, lower is the probability of outage over the entire link. Therefore STFS and STS-IM offer quite a striking flexibility between diversity and performance gain.

In Figs. 7 and 8, outage probability is plotted as functions of receive antennas at the IoT gateway (number of antennas at the application endpoint is kept fixed at 8) and at the endpoint (number of antennas at the gateway is kept fixed at 32) respectively. Increase in the number of receive antennas at the gateway have profound effect on the outage probability as compared with increase in number of antennas at the endpoint. As the gateway is equipped with large number of antennas, the outage probability drops dramatically and converge to almost null both for the indoor and outdoor scenarios. Increasing the number of antennas at the endpoint do not help that much. The outage performance drops a bit but saturates after the number of antennas increases more than 30 .

\section{Open Challenges And Future Direction}

We make the first-ever attempt to develop an end-to-end framework that involves a real-time digital dashboard for collecting and categorizing population at different levels of risk of experiencing anxiety attack. Based on the observation at the dashboard, appropriate action is initiated to respond to the requirement of the individual as well as a particular geographical area. Initial simulation results based on the developed system prototype and communication link design reveal that the framework offers real-time insight into the status of the population's mental health with high reliability in an energy-efficient way. However, there are several research issues that need to be addressed and interesting generalizations that need to be added to the framework in order to develop it into a full-fledged application.

A major challenge is to validate the applicability of our proposed framework to enjoy its benefits in full potential. This can only be done by conducting real-world clinical trials. The wearable IoT devices have to be distributed to people based on their willingness to participate in the study. With proper ethical consent from the participants, the system can be evaluated in practical scenarios.

Right now, anxiety index is measured using real-time streamed data from the users and then plotted on the dashboard. The framework is, therefore, only able to estimate risk of anxiety in the present timeline. However, in future, it should be able to predict the risk of anxiety attack for the patient. Towards that end, the framework needs to incorporate neural networks and machine learning algorithms for forecasting the risk of anxiety attack.

In order to bring the proposed framework to reality, mobile application and fully-featured cloud-based digital dashboard need to be developed in practicality. Cloud hosting service needs to be availed from the providers, like Microsoft, Azure Suite, AWS Cloud, Google Cloud and Ubidots. For generalization of the dashboard, the categorization needs to include more factors like demographic attributes of gender, age and 
fitness level. The lifestyle factors can also be incorporated by taking into account issues like drinking water contamination, manipulation and poor air quality.

\section{CONCLUSION}

In this article, we have proposed a real-time IoT-based digital dashboard to categorize populations' anxiety risk levels based on their physiological data and geographic location. The proposed architecture can be regarded as the next-generation anxiety risk classification mean for the healthcare industry 4.0 as it is capable of generating automated actions through analytics on the collected data and predefined thresholds. A detailed communication architecture for data transmission from wearable sensors to a cloud has been presented. Moreover, a high-level prototype was developed using the NodeMCU platform, a pulse oximetry sensor, a wrist blood pressure monitor, and an OLED screen displaying Google Sheets. In addition, the integration of the emerging binding technologies has been shown to improve the energy performance of the proposed architecture. Finally, we showed that the availability of a centralized dashboard providing real-time insight about the mental health status of a population is expected to help authorities effectively tackle any pandemic in the future.

\section{REFERENCES}

[1] L. Tan, "Preventing the transmission of covid-19 amongst healthcare workers," Journal of Hospital Infection, vol. 105, no. 2, pp. 364-365, 2020.

[2] O. Singh et al., "Mental health of migrant laborers in covid-19 pandemic and lockdown: Challenges ahead," Indian Journal of Psychiatry, vol. 62, no. 3, p. 233, 2020.

[3] C. Wang, R. Pan, X. Wan, Y. Tan, L. Xu, C. S. Ho, and R. C. Ho, "Immediate psychological responses and associated factors during the initial stage of the 2019 coronavirus disease (covid-19) epidemic among the general population in china," International journal of environmental research and public health, vol. 17, no. 5, p. 1729, 2020.

[4] J. Zhou, H. Zogan, S. Yang, S. Jameel, G. Xu, and F. Chen, "Detecting community depression dynamics due to covid-19 pandemic in australia," IEEE Transactions on Computational Social Systems, 2021.

[5] M. Pierce, H. Hope, T. Ford, S. Hatch, M. Hotopf, A. John, E. Kontopantelis, R. Webb, S. Wessely, S. McManus et al., "Mental health before and during the covid-19 pandemic: a longitudinal probability sample survey of the uk population," The Lancet Psychiatry, vol. 7, no. 10, pp. 883-892, 2020.

[6] M. T. Sinnard, C. R. Raines, and S. L. Budge, "The association between geographic location and anxiety and depression in transgender individuals: an exploratory study of an online sample," Transgender Health, vol. 1, no. 1, pp. 181-186, 2016.

[7] M. Javaid, A. Haleem, R. Vaishya, S. Bahl, R. Suman, and A. Vaish, "Industry 4.0 technologies and their applications in fighting covid19 pandemic," Diabetes \& Metabolic Syndrome: Clinical Research \& Reviews, vol. 14, no. 4, pp. 419-422, 2020.

[8] K. T. Kadhim, A. M. Alsahlany, S. M. Wadi, and H. T. Kadhum, "An overview of patient's health status monitoring system based on internet of things (iot)," Wireless Personal Communications, vol. 114, pp. 22352262,2020

[9] D. Concannon, K. Herbst, and E. Manley, "Developing a data dashboard framework for population health surveillance: widening access to clinical trial findings," JMIR formative research, vol. 3, no. 2, p. e11342, 2019.

[10] T. Perumal, M. N. Sulaiman, and C. Y. Leong, "Internet of things (iot) enabled water monitoring system," in 2015 IEEE 4th Global Conference on Consumer Electronics (GCCE), 2015, pp. 86-87.

[11] M. Zhou, A. M. Abdulghani, M. A. Imran, and Q. H. Abbasi, "Internet of things (iot) enabled smart indoor air quality monitoring system," in Proceedings of the 2020 International Conference on Computing, Networks and Internet of Things, 2020, pp. 89-93.

[12] E. M. Katemboh, R. Abdulla, V. Jayapal, S. K. Selvaperumal, and D. Ratnadurai, "Integrated animal health care using iot," 2019.
[13] S. Siddagangaiah, "A novel approach to iot based plant health monitoring system," Int. Res. J. Eng. Technol, vol. 3, no. 11, pp. 880-886, 2016.

[14] J. Cabra, D. Castro, J. Colorado, D. Mendez, and L. Trujillo, "An iot approach for wireless sensor networks applied to e-health environmental monitoring," in 2017 IEEE International Conference on Internet of Things (iThings) and IEEE Green Computing and Communications (GreenCom) and IEEE Cyber, Physical and Social Computing (CPSCom) and IEEE Smart Data (SmartData), 2017, pp. 578-583.

[15] P. Pace, G. Aloi, G. Caliciuri, R. Gravina, C. Savaglio, G. Fortino, G. Ibáñez-Sánchez, A. Fides-Valero, J. Bayo-Monton, M. Uberti et al., "Inter-health: An interoperable iot solution for active and assisted living healthcare services," in 2019 IEEE 5th World Forum on Internet of Things (WF-IoT), 2019, pp. 81-86.

[16] W. M. Shah, M. H. Yaakob, N. Harum, A. Hassan, M. F. I. Othman, I. R. A. Hamid et al., "Internet of things based heart rate monitoring and alert system," JACTA, vol. 2, no. 1, pp. 27-31, 2020.

[17] E. C. Wick, L. Pierce, M. C. Conte, and J. A. Sosa, "Operationalizing the operating room: ensuring appropriate surgical care in the era of covid-19," Annals of surgery, vol. 272, no. 2, p. e165, 2020.

[18] K. Thorlund, L. Dron, J. Park, G. Hsu, J. I. Forrest, and E. J. Mills, "A real-time dashboard of clinical trials for covid-19," The Lancet Digital Health, vol. 2, no. 6, pp. e286-e287, 2020.

[19] J. J. Reeves, H. M. Hollandsworth, F. J. Torriani, R. Taplitz, S. Abeles, M. Tai-Seale, M. Millen, B. J. Clay, and C. A. Longhurst, "Rapid response to covid-19: health informatics support for outbreak management in an academic health system," Journal of the American Medical Informatics Association, vol. 27, no. 6, pp. 853-859, 2020.

[20] S. Gao, J. Rao, Y. Kang, Y. Liang, and J. Kruse, "Mapping county-level mobility pattern changes in the united states in response to covid-19," SIGSpatial Special, vol. 12, no. 1, pp. 16-26, 2020.

[21] R. A. Dixit, S. Hurst, K. T. Adams, C. Boxley, K. Lysen-Hendershot, S. S. Bennett, E. Booker, and R. M. Ratwani, "Case report: Rapid development of visualization dashboards to enhance situation awareness of covid-19 telehealth initiatives at a multi-hospital healthcare system," Journal of the American Medical Informatics Association, 2020.

[22] I. Dey, H. Joshi, and N. Marchetti, "Space-time spreading aided distributed MIMO-WSNs," IEEE Communications Letters, vol. 25, no. 4, pp. 1338-1342, 2021

[23] Y. Zhan and F. Huang, "Generalized spatial modulation with multi-index modulation," IEEE Communications Letters, vol. 24, no. 3, pp. 585-588, 2020.

[24] K. Haneda and et. al., "5g 3gpp-like channel models for outdoor urban microcellular and macrocellular environments," in 2016 IEEE $83 \mathrm{rd}$ Vehicular Technology Conference (VTC 2016-Spring), 2016, p. 7 pages.

[25] K. Haneda, L. Tian, H. Asplund, J. Li, Y. Wang, D. Steer, C. Li, T. Balercia, S. Lee, Y. Kim et al., "Indoor 5g 3gpp-like channel models for office and shopping mall environments," in 2016 IEEE International Conference on Communications Workshops (ICC). IEEE, 2016, pp. 694-699. 